\title{
Persistence of Tripartite Nonlocality for Non-inertial Observers
}

\author{
Alexander Smith and Robert B. Mann ${ }^{1}$ \\ ${ }^{1}$ Department of Physics, University of Waterloo, Waterloo, Ontario Canada N2L 3G1
}

\begin{abstract}
We consider the behaviour of bipartite and tripartite non-locality between fermionic entangled states shared by observers, one of whom uniformly accelerates. We find that while fermionic entanglement persists for arbitrarily large acceleration, the Bell/CHSH inequalities cannot be violated for sufficiently large but finite acceleration. However the Svetlichny inequality, which is a measure of genuine tripartite non-locality, can be violated for any finite value of the acceleration.
\end{abstract}

The relationship between entanglement and nonlocality is at the root of the foundations of quantum mechanics. Bell-type inequalities 1, such as the ClauserHorner-Shimony-Holt (CHSH) inequality 2, place an upper bound on the correlations compatible with classical local realistic (or hidden variable) theories. All pure entangled states of two qubits violate the CHSH inequality, with the amount of violation increasing with the degree of bipartite entanglement [3]. For pure tripartite-entangled states the Svetlichny inequality 4 functions in a similar way - its violation is a sufficient condition for the confirmation of genuine three-qubit nonlocal correlations. It has recently been shown that a class of generalized GHZ (GGHZ) states do not violate the Svetlichny inequality if their 3 -tangle is less than $1 / 2$, whereas another class of states known as maximal slice (MS) states violate the Svetlichny inequality with the amount of violation increasing with the degree of tripartite entanglement [5].

In this paper we investigate the behaviour of nonlocal correlations of bipartite and tripartite entangled states shared between observers in a situation where one observer moves with uniform acceleration. Previous work has shown that both bipartite [6, 7] and tripartite [8, 9] entanglement are degraded as a function of increasing acceleration of a given detector. For fermionic states this degradation persists to a finite value [7, 9] in the large acceleration limit in both cases. We find that whereas bipartite nonlocal correlations vanish for finite values of the acceleration, tripartite nonlocal correlations persist for any finite acceleration for both GGHZ and MS threequbit states provided the respective control parameters are appropriately chosen, vanishing only in the infinite acceleration limit. This demonstrates that tripartite entanglement and its associated nonlocal correlations are more robust to relativistic effects.

We consider a flat spacetime in which there is a uniformly accelerating observer, Rob. The two other observers, Alice and Charlie, remain inertial. Each observes the same state of a fermionic field (described by a Grassmann scalar, the simplest case preserving the essential Dirac characteristics [10]). However while both Charlie and Alice use a basis of Minkowski modes (plane waves in the massless case) for the description of their respective parts of the field state, Rob uses the so-called Rindler modes [11. Uniformly accelerated detectors cou- ple to these modes. The change of basis between Rindler and Minkowski modes (given by the so-called Bogoliubov coefficients [11, 12]) mixes Minkowski creation and annihilation operators. Consequently the Minkowski and Rindler vacua are not the same, giving rise to the wellknown Unruh effect [13].

Rindler coordinates $(\eta, \xi, y, z)$ describe a family of observers with uniform acceleration $a$ and divide Minkowksi spacetime (with coordinates $(t, x, y, z)$ ) into 4 regions separated by a light cone centered on the origin and labelled clockwise as regions F, I, P, and II; Rightward accelerating observers are located in region I (where $t=\xi \sinh (a \eta / c)$ and $x=\xi \cosh (a \eta / c))$ and are causally disconnected from their analogous counterparts in region II. Monochromatic solutions of the field equation relevant to the accelerating detector in Rindler coordinates are called Rindler modes. The annihilation operator in region I for the Rindler mode of a given particle species of frequency $\omega$, and spin- $\sigma$ component is denoted $c_{\omega, \sigma, I}$, with its antiparticle counterpart denoted $d_{\omega, \sigma, I}$, and its creation operators obtained from the dagger of these. Region II has corresponding pairs of annihilation/creation operators. From the accelerated observer's viewpoint the Hilbert space factorizes as $\mathcal{H}_{I} \otimes \mathcal{H}_{I I}$. Since Rob (by definition) is confined to region I, we must trace out the part of the state outside of this region (ie we trace over all states in region $\mathcal{H}_{I I}$ ), since he cannot observe it. Analogous considerations apply to observers in region II, generally referred to as anti-Rob [7].

An inertial observer would express Rob's vacuum mode $|0\rangle_{R}$, and first excitation of his vacuum mode $|1\rangle_{R}$ as $[7]$

$$
\begin{aligned}
|0\rangle_{R} & =\cos r|0\rangle_{I}|0\rangle_{I I}+\sin r|1\rangle_{I}|1\rangle_{I I}, \\
|1\rangle_{R} & =|1\rangle_{I}|0\rangle_{I I} .
\end{aligned}
$$

where $|0\rangle_{I}$ and $|1\rangle_{I}$ are respectively the Minkowski vacuum and first excitation of the Minkowski vacuum in region I; $|0\rangle_{I I}$ and $|1\rangle_{I I}$ are defined similarly over region II. For simplicity we consider all orthogonal modes as unexcited. The parameter $r$ is

$$
\cos r=\frac{1}{\sqrt{1+\exp (-2 \pi \Omega)}},
$$

where $\Omega=\frac{\omega c}{a}$ and $a$ is Rob's acceleration, $c$ is the speed of light and $\omega$ is the central frequency of the fermion 
wave packet. Note that $r=0$ corresponds to an inertial observer and $r=\pi / 4$ corresponds to an observer in the infinite acceleration limit.

It is possible to construct a different set of modes that have the property that they are linear combinations of purely positive-frequency Minkowski modes. Referred to as Unruh modes, they are given by [11]

$$
\begin{aligned}
& \tilde{c}_{\omega, \sigma, R}^{\dagger}=\cos r c_{\omega, \sigma, I}^{\dagger}-\sin r d_{\omega,-\sigma, I I}^{\dagger} \\
& \tilde{c}_{\omega, \sigma, L}^{\dagger}=\cos r c_{\omega, \sigma, I I}^{\dagger}-\sin r d_{\omega,-\sigma, I}^{\dagger}
\end{aligned}
$$

where $\tan r=e^{-\pi \Omega}$. The subscripts $\mathrm{L}$ and $\mathrm{R}$ denote left and right modes, and are related to each other by an exchange of regions I and II. In terms of creation and annihilation operators, this means that (4) can be rewritten as a linear combination of only Minkowski creation operators, implying that the Minkowski and Unruh vacua are the same.

Hence we can write the Minkowski vacuum $|0\rangle_{M}$ as $|0\rangle_{M}=\bigotimes_{\omega}|0\rangle_{\omega, U}$ where $\tilde{c}_{\omega, \sigma, R}^{\dagger}$ and $\tilde{c}_{\omega, \sigma, L}^{\dagger}$ each annihilate $|0\rangle_{\omega, U}$ for every $\sigma$. We can therefore restrict our considerations to a particular frequency $\omega$. An arbitrary Unruh excitation is

$$
|1\rangle_{\omega, U}=\left(q_{R} \tilde{c}_{\omega, \sigma, R}^{\dagger}+q_{L} \tilde{c}_{\omega, \sigma, L}^{\dagger}\right)|0\rangle_{\omega, U}
$$

with $\left|q_{R}\right|^{2}+\left|q_{L}\right|^{2}=1$. We shall set $q_{L}=0$, (the socalled single mode approximation [14]), and henceforth drop the redundant subscripts $\omega, U$, and $\sigma$.

The well-known CHSH inequality [2]

$$
\left|C(\mathbf{a}, \mathbf{b})+C\left(\mathbf{a}^{\prime}, \mathbf{b}\right)+C\left(\mathbf{a}, \mathbf{b}^{\prime}\right)-C\left(\mathbf{a}^{\prime}, \mathbf{b}^{\prime}\right)\right| \leq 2
$$

quantifies bipartite non-locality, where $\mathbf{a}$ and $\mathbf{a}^{\prime}$ are a set of unit vectors belonging to one observer and $\mathbf{b}$ and $\mathbf{b}^{\prime}$ are unit vectors belonging to another observer who are measuring the spin along these directions. The correlation functions $C(\mathbf{a}, \mathbf{b})$ are given by

$$
C(\mathbf{a}, \mathbf{b})=\left(\frac{2}{\hbar}\right)^{2}\left\langle\psi\left|\mathbf{a} \cdot S_{1} \otimes \mathbf{b} \cdot S_{2}\right| \psi\right\rangle .
$$

where $S_{1}$ and $S_{2}$ are the spin operators acting on modes 1 and 2 respectively. Let us consider the singlet state

$$
|\psi\rangle=\frac{1}{\sqrt{2}}(|10\rangle-|01\rangle)
$$

invariant under the unitary operators which generate rotations of coordinate systems. This means that $C(\mathbf{a}, \mathbf{b})$ is a function of $\cos \theta_{a b}:=\mathbf{a} \cdot \mathbf{b}$ only, and hence there is no loss of generality in assuming that a points along the $z$ axis and that $\mathbf{b}$ lies in the $x-z$ plane. Then equation (7) becomes

$$
C(\mathbf{a}, \mathbf{b})=\left\langle\psi\left|\sigma_{1 z} \otimes\left(\sigma_{2 z} \cos \theta_{a b}+\sigma_{2 x} \sin \theta_{a b}\right)\right| \psi\right\rangle .
$$

An accelerating observer making measurements in a sufficiently small interval about $\eta=0$ will use the same vectors as in eq. (7), but would see qubit 2 described by equations (1) and 22). Constructing the reduced density operator $\rho_{A, I}=\operatorname{tr}_{I I}[|\psi\rangle\langle\psi|]$ by tracing out region II we obtain from $(9)$

$$
C(\mathbf{a}, \mathbf{b})=-\frac{1}{2}(1+\cos 2 r) \cos \theta_{a b} .
$$

Let us now restrict our attention to the special case in which (i) the vectors $\mathbf{a}, \mathbf{a}^{\prime}, \mathbf{b}, \mathbf{b}^{\prime}$ are co-planar; (ii) $\mathbf{a}$ and $\mathbf{b}$ are parallel; and (iii) $\theta_{a b^{\prime}}=\theta_{a^{\prime} b}=\gamma$. Then the CHSH inequality will be satisfied provided

$$
\cos ^{2} r\left|\sin ^{2} \gamma+\cos \gamma\right| \leq 1
$$

yielding a specific threshold acceleration $a_{t}=\frac{2 \pi \omega c}{\ln 4}$ above which the CHSH inequality cannot be violated, despite the persistence of fermionic entanglement for $a>a_{t}[7]$.

Turning next to tripartite nonlocality, we consider the generalized GHZ (GGHZ) state $\left|\psi_{g}\right\rangle$ and the maximal slice (MS) state $\left|\psi_{s}\right\rangle$

$$
\begin{aligned}
\left|\psi_{g}\right\rangle & =\cos \theta_{1}|000\rangle+\sin \theta_{1}|111\rangle \\
\left|\psi_{s}\right\rangle & =\frac{1}{\sqrt{2}}\left\{|000\rangle+|11\rangle\left[\cos \theta_{3}|0\rangle+\sin \theta_{3}|1\rangle\right]\right\}
\end{aligned}
$$

where $\theta_{1}$ and $\theta_{3}$ are parameters controlling the entanglement of the state. The usual GHZ state is realized by setting $\theta_{1}=\pi / 4$ in 12 or by setting $\theta_{3}=\pi / 2$ in $(13)$. To avoid difficulties in distinguishing violations arising from 2-body versus 3-body entanglement [5, 15, 16, we shall consider the Svetlichny inequality, as its violation is a sufficient condition for the confirmation of genuine 3-qubit nonlocal correlations 4, 5. This inequality emerged from a consideration of a model of nonlocal-local-realism [4, where two of the qubits are nonlocally correlated but are locally correlated to the third, implying an entanglement between the three qubits that cannot be decomposed into a case of familiar two body entanglement.

Suppose we have a system that can be partitioned into three subsystems, respectively detectable by Alice, Charlie and Bob. Alice performs the measurements $A=\hat{\mathbf{a}} \cdot \vec{\sigma}_{1}$ or $A^{\prime}=\hat{\mathbf{a}}^{\prime} \cdot \vec{\sigma}_{1}$ on system 1 , Charlie performs $C=\hat{\mathbf{c}} \cdot \vec{\sigma}_{2}$ or $C^{\prime}=\hat{\mathbf{c}}^{\prime} \cdot \vec{\sigma}_{2}$ on system 2 and Bob performs measurements $B=\hat{\mathbf{b}} \cdot \vec{\sigma}_{3}$ or $B^{\prime}=\hat{\mathbf{b}}^{\prime} \cdot \vec{\sigma}_{3}$ on system 3 , where $\vec{\sigma}_{i}$ are projection operators that can be expressed in terms of the Pauli operators and $\hat{\mathbf{a}}, \hat{\mathbf{a}}^{\prime}, \hat{\mathbf{b}}, \hat{\mathbf{b}}^{\prime}, \hat{\mathbf{c}}, \hat{\mathbf{c}}^{\prime}$ are unit vectors corresponding to the direction of measurement. For a theory consistent with Svetlichny's nonlocal-local-realism

$$
S(\Psi):=|\langle\psi|S| \psi\rangle| \leq 4
$$

where $S$ is the Svetlichny operator defined as

$$
S=A\left(C K+C^{\prime} K^{\prime}\right)+A^{\prime}\left(C K-C^{\prime} K^{\prime}\right) .
$$

where $K=B+B^{\prime}$ and $K^{\prime}=B-B^{\prime}$.

To maximize the expectation value of $S$ for the the GGHZ and MS states [5], we set $\mathbf{a}=$ 
$\left(\sin \theta_{a} \cos \phi_{a}, \sin \theta_{a} \sin \phi_{a}, \cos \theta_{a}\right)$ and similarly for $\hat{\mathbf{a}}^{\prime}, \hat{\mathbf{b}}$, $\hat{\mathbf{b}}^{\prime}, \hat{\mathbf{c}}$ and $\hat{\mathbf{c}}^{\prime}$. Defining unit vectors $\hat{\mathbf{d}}$ and $\hat{\mathbf{d}}^{\prime}$ such that $\hat{\mathbf{c}}+\hat{\mathbf{c}}^{\prime}=2 \hat{\mathbf{d}} \cos \theta$ and $\hat{\mathbf{c}}-\hat{\mathbf{c}}^{\prime}=2 \hat{\mathbf{d}} \sin \theta$, with $D=\hat{\mathbf{d}} \cdot \vec{\sigma}_{2}$ and $D^{\prime}=\hat{\mathbf{d}}^{\prime} \cdot \vec{\sigma}_{2}$, the expectation value of 15 is

$$
\begin{gathered}
S(\psi)=2 \mid \cos \theta\left(\langle A D B\rangle-\left\langle A^{\prime} D B^{\prime}\right\rangle\right) \\
+\sin \theta\left(\left\langle A D^{\prime} B^{\prime}\right\rangle+\left\langle A^{\prime} D^{\prime} B\right\rangle\right) \mid \\
\leq 2 \mid\left(\langle A D B\rangle^{2}+\left\langle A D^{\prime} B^{\prime}\right\rangle^{2}\right)^{1 / 2} \\
+\left(\left\langle A^{\prime} D^{\prime} B\right\rangle^{2}+\left\langle A^{\prime} D B^{\prime}\right\rangle^{2}\right)^{1 / 2} \mid
\end{gathered}
$$

using the identity $x \cos \theta+y \sin \theta \leq\left(x^{2}+y^{2}\right)^{1 / 2}$.

Replacing Bob by the uniformly accelerating Rob, who observes qubit 3 in a sufficiently small interval about $\eta=$ 0 , we construct the density operator, $\rho=|\psi\rangle\langle\psi|$, from (1) and (2) and use this to evaluate (16), yielding

$$
\begin{aligned}
S\left(\psi_{g}\right) & \leq 2\left\{\left(2 \cos ^{2} \theta_{1} \cos ^{2} r-1\right)^{2}\left(\cos ^{2} \theta_{d}+\cos ^{2} \theta_{d^{\prime}}\right) \cos ^{2} \theta_{a}+\sin ^{2} 2 \theta_{1} \cos ^{2} r\left(\sin ^{2} \theta_{d}+\sin ^{2} \theta_{d^{\prime}}\right)\right\}^{1 / 2} \sin ^{2} \theta_{a} \\
& +2\left\{\left(2 \cos ^{2} \theta_{1} \cos ^{2} r-1\right)^{2}\left(\cos ^{2} \theta_{d}+\cos ^{2} \theta_{d^{\prime}}\right) \cos ^{2} \theta_{a^{\prime}}+\sin ^{2} 2 \theta_{1} \cos ^{2} r\left(\sin ^{2} \theta_{d}+\sin ^{2} \theta_{d^{\prime}}\right) \sin ^{2} \theta_{a^{\prime}}\right\}^{1 / 2}
\end{aligned}
$$

for the GGHZ state (12).

Maximizing with respect to $\theta_{a}$ and $\theta_{a^{\prime}}$ and noting that the maxima of $\cos ^{2} \theta_{d}+\cos ^{2} \theta_{d^{\prime}}$ and $\sin ^{2} \theta_{d}+\sin ^{2} \theta_{d^{\prime}}$ are respectively 1 and 2 yields

$$
S\left(\psi_{g}\right) \leq\left\{\begin{array}{ll}
4\left(2 \cos ^{2} \theta_{1} \cos ^{2} r-1\right) & : C_{3} \geq C_{4} \\
4 \sqrt{2} \sin 2 \theta_{1} \cos r & : C_{3}<C_{4}
\end{array} .\right.
$$

where

$$
C_{3}=\left(2 \cos ^{2} \theta_{1} \cos ^{2} r-1\right)^{2} \quad C_{4}=\sin ^{2} 2 \theta_{1} \cos ^{2} r
$$

As $r \rightarrow 0$ Rob, the accelerated observer, becomes the inertial observer Bob and eq. 18 agrees with the results of [5]. We plot in figure 1 the value of $S$ when the inequality in (18) is saturated as a function of the control parameter $\theta_{1}$ and Rob's acceleration $r$. A straightforward (but lengthy) calculation of the $\pi$-tangle (a measure of tripartite entanglement for mixed states [9, 17]) indicates that $\theta_{1}$ monotonically increases as a function of the $\pi$-tangle. Hence we find for all finite values of the acceleration parameter that nonlocality increases as a function of increasing entanglement for the GGHZ state beyond a certain threshold value of the $\pi$-tangle. Note that more entanglement is required to violate the Svetlichny inequality as $a$ increases; it is not possible to violate the inequality in the infinite acceleration limit.

For the MS state its reduced symmetry between only qubits 1 and 2 implies that there are two cases to consider for one accelerating observer: (i) when Rob is measuring either qubit 1 or 2 and (ii) when Rob is measuring qubit 3.

For case (i) we construct the density operator by substituting equations (1) and (2) into the second qubit of the MS state in equation (13). Tracing out region II to obtain the density operator $\rho_{A I C}$ and using this to eval-

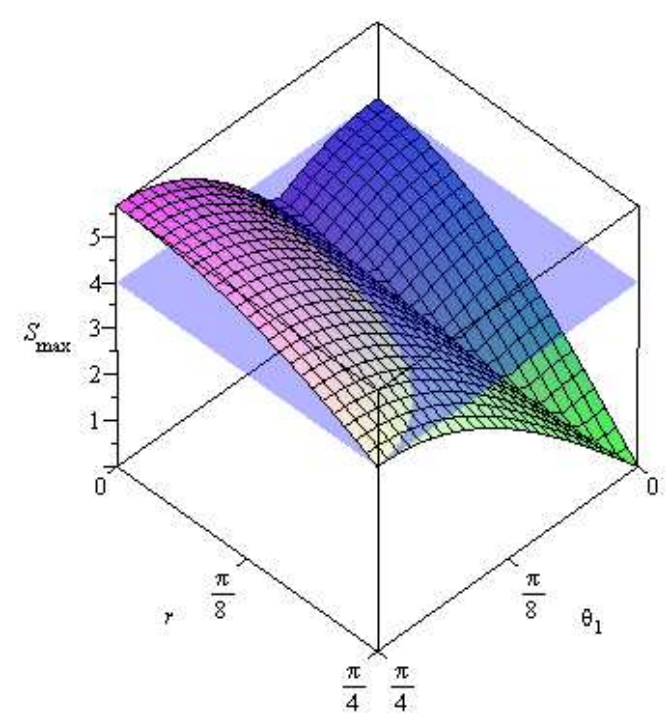

FIG. 1: The maximal value of the left hand side of the Svetlichny inequality plotted against Rob's acceleration $r$ and the control parameter $\theta_{1}$ for the GGHZ state. As $\theta_{1}$ increases so does the $\pi$-tangle of the state.

uate eq. (16) gives

$$
S\left(\psi_{s}\right) \leq 4 \cos r\left\{\cos ^{2} \theta_{3}+2 \sin ^{2} \theta_{3}\right\}^{1 / 2}
$$

where $S\left(\psi_{s}\right)$ has a maximum at $\phi_{d}-\phi_{d^{\prime}}=\theta_{d}=\theta_{d^{\prime}}=\pi / 2$ [5. The maximal value of $S\left(\psi_{s}\right)$ from eq. (20) is plotted in figure 2. A calculation indicates that $\theta_{3}$ monotonically increases as a function of the associated $\pi$-tangle. In this case we see that for any finite value of the acceleration there exists a value of the $\pi$-tangle (or $\theta_{3}$ ) for which the maximal value of $S\left(\psi_{s}\right)$ is larger than 4 and the Svetlichny inequality is violated. 


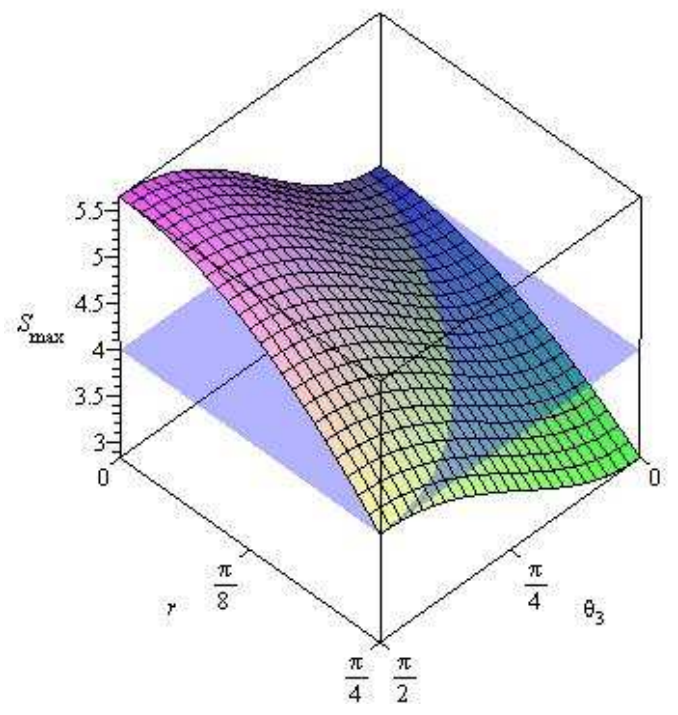

FIG. 2: The maximal value of $S\left(\psi_{M}\right)$ plotted as a function of the control parameter $\theta_{3}$ and Rob's acceleration $r$, with Rob measuring qubit 2 .

For case (ii) the analogous result is

$$
S\left(\psi_{s}\right) \leq 4\left\{\cos ^{2} \theta_{3} \cos ^{2} 2 r+2 \sin ^{2} \theta_{3} \cos ^{2} r\right\}^{1 / 2}
$$

and it is straightforward to show that the maximal value of $S\left(\psi_{s}\right)$ is also larger than 4 for all finite values of $r$ provided $\theta_{3}$ is appropriately chosen.

For increasing uniform acceleration, violation of both the bipartite Bell-CHSH and tripartite Svetlichny inequalities becomes increasingly difficult, despite the survival of fermionic entanglement in the large $a$ limit. For $a>a_{t}$ all bipartite nonlocality vanishes, whereas tripartite nonlocality persists for all finite values of $a$. This suggests a fundamental difference between tripartite and bipartite entanglement in relativistic settings.
R.B.M was supported in part by the Natural Sciences and Engineering Research Council of Canada.

Note added As this work was being completed reference 18 appeared, in which similar results were obtained for the Bell/CHSH inequalities.

[1] J. S. Bell, Physics 1, 195 (1964).

[2] J. F. Clauser et al., Phys. Rev. Lett. 23, 880 (1969).

[3] N. Gisin, Phys. Lett. A 154, 201 (1991); S. Popescu and D. Rohrlich, Phys. Lett. A 166, 293 (1992).

[4] G. Svetlichny Phys. Rev., D35 3066 (1987).

[5] S. Debnath, P. Rungta, S. Ghose, N. Sinclair and R. Stock. Phys. Rev. Lett., 102: 250404 (2009).

[6] I. Fuentes-Schuller and R. B. Mann. Phys. Rev. Lett. 95: 120404 (2005)

[7] R. B. Mann P. M. Alsing, I. Fuentes-Schuller and T. E. Tessier, Phys. Rev. A74: 032326 (2006).

[8] M. Hwang, D. Park, and E. Jung, Phys. Rev. A 83, 012111 (2011)

[9] J. Wang and J. Jing, Phys. Rev. A 83, 022314 (2011)

[10] E. Martn-Martnez and I. Fuentes, Phys. Rev. A83, 052306 (2011)

[11] D. E. Bruschi, J. Louko, E. Martn-Martnez, A. Dragan,and I. Fuentes, Phys. Rev. A 82: 042332 (2010).

[12] R. Jauregui, M. Torres, and S. Hacyan, Phys. Rev. D43,3979 (1991); P. Langlois, Phys. Rev. D70: 104008 (2004).

[13] W. G. Unruh, Phys. Rev. D14, 870 (1976).

[14] P. M. Alsing and G. J. Milburn, Phys. Rev. Lett. 91,180404 (2003); P. M. Alsing, D. McMahon, and G. J. Milburn, J. Opt.B: Quantum Semiclass. Opt. 6, S834 (2004).

[15] D. Collins Phys. Rev. Lett. 88:170405 (2002).

[16] J. L. Cereceda Phys. Rev., A66:024102 (2002).

[17] Y. U. Ou and H. Fan. Phys. Rev., A75: 062308 (2007).

[18] N. Friis, P. Kohler, E. Martn-Martnez, and R. A. Bertlmann, arXiv:1107.3235 